\title{
Electromagnetic wave polarization state evolution in weakly anisotropic and nonuniform media with dissipation
}

\author{
B. Bieg, J. Chrzanowski \\ Institute of Physics, Maritime University of Szczecin, Waly Chrobrego 1-2, 72-500 Szczecin, Poland
}

Received August 26, 2017; accepted September 28, 2017; published September 30, 2017

\begin{abstract}
The change of the polarization state is analysed of an electromagnetic beam propagating in weakly anisotropic and smoothly inhomogeneous media with dissipation. On the basis of a quasi-isotropic approximation, which provides a consequent asymptotic solution of Maxwell's equation, a differential equation for the evolution of a fourcomponent Stokes vector is derived. The obtained equation generalizes the previous results for nonadsorbing media and is written in terms of a dielectric tensor of birefringent media with dissipation. The formalism is illustrated by an example of magnetised plasma with dissipation due to electron collisions.
\end{abstract}

Traditional methods for the analysis of the polarization state of an electromagnetic wave passing through nonuniform birefringent media have been concentrated on the case of negligible wave absorption. In such a case the polarization state evolution is limited to a combination of polarization plane rotation (the Faraday effect in media with circular birefringence) and change in the ellipticity state (Cotton-Mouton effect in media with linear birefringence). To describe such evolution there are two main approaches. The first one deals with coupled wave equations for the components of an electromagnetic wave field. In stratified media it is Budden's method [1-2], whereas the modification of Budden's approach for an arbitrarily inhomogeneous medium is a quasi-isotropic approximation (QIA) of geometric optics [3-6]. The main disadvantage of QIA is the fact that for an electromagnetic wave it describes the evolution of amplitude and phase of each perpendicular component of an electric field whereas from polarimetric measurements information is obtained on the amplitude of both field components and only their phase difference. The second, alternative approach - the Stokes vector formalism (SVF) for the electromagnetic wave in stratified media, initiated in [7] and reviewed in [8], was applied for purposes of e.g. fiber and plasma polarimetry. In the case of a medium without dissipation, SVF - based on the reduced threecomponent Stokes vector $\mathbf{S}$ is completely sufficient.

The purpose of this work is to derive an evolution equation for the full four-component Stokes vector directly from the QIA. The obtained Stokes vector equation generalizes the previous results for refractionless and nonadsorbing media. The paper is organized as follows. At the beginning, basic QIA equations are

*E-mail: b.bieg@am.szczecin.pl presented. Next, equations for the four-component Stokes vector are derived from the QIA for a weakly anisotropic medium of a general type. Then, a general theory is applied for Stokes-vector evolution in weakly anisotropic collisional plasma, such ionosphere, laboratory or tokamak plasma with parameters typical for modern tokamak machines.

The theory of electromagnetic wave propagation in weakly anisotropic media was developed in [3] in the form of a quasi-isotropic approximation of the geometric optics method. A short outline of the QIA is presented in the books [4-5] and in the review paper [6]. The quasiisotropic approximation of geometrical optics is efficient for describing the evolution of electromagnetic wave components in a 3D weakly inhomogeneous weakly anisotropic medium. The dielectric permittivity $\varepsilon_{i j}$ of a weakly anisotropic medium could be split into two parts: a large isotropic component $\varepsilon_{0} \delta_{i j}$, where $\delta_{i j}$ is the unit vector, and an anisotropic component $v_{i j}$, which is small as compared with the isotropic part $\varepsilon_{0}$ : $\max \left|v_{i j}\right| \ll \varepsilon_{0}$ :

$$
\varepsilon_{i j}=\varepsilon_{0} \delta_{i j}+v_{i j}
$$

The quantity $\mu_{a}=\max \left|v_{i j}\right| / \varepsilon_{0} \ll 1$ is an "anisotropic" small addition to the traditional small parameter of geometrical optics $\mu_{G O}=1 / \mathrm{kL} \ll 1$, where $k$ is the wave number and $L$ is the characteristic scale of an inhomogeneous medium. Thy QIA comes from the solution of Maxwell's equations by asymptotic expansion of the electromagnetic wave field $\mathbf{E}$ with respect to the combined small parameter $\mu=\max \left(\mu_{G O}, \mu_{a}\right)$. In the lowest order of the QIA, the monochromatic electromagnetic wave field $\mathbf{E}$ has the form of a transverse wave:

$$
\mathbf{E}=E_{x} \boldsymbol{e}_{x}+E_{y} \boldsymbol{e}_{y}=A\left(\Gamma_{x} \boldsymbol{e}_{x}+\Gamma_{y} \boldsymbol{e}_{y}\right) \exp [i k \Psi]
$$

In the frame of the QIA theory a weak anisotropy influences the polarization vector $\boldsymbol{\Gamma}=\Gamma_{x} \boldsymbol{e}_{x}+\Gamma_{y} \boldsymbol{e}_{y}$ rather than the eikonal $\Psi$ and the wave amplitude $A$ : the latter obey the same equations as in the isotropic medium. The polarization vector components $\Gamma_{x}$ and $\Gamma_{y}$ obey the QIA 
coupled equations, which can be presented in the form [6]:

$$
\left\{\begin{array}{l}
\Gamma_{x}^{\prime}=\frac{1}{2} i k \varepsilon_{0}^{-1 / 2}\left(v_{11} \Gamma_{x}+v_{12} \Gamma_{y}\right), \\
\Gamma_{y}^{\prime}=\frac{1}{2} i k \varepsilon_{0}^{-1 / 2}\left(v_{21} \Gamma_{x}+v_{22} \Gamma_{y}\right),
\end{array}\right.
$$

where derivatives are calculated over $\mathrm{d} z$ - the elementary arc length of the ray.

The four-component Stokes vector $\boldsymbol{S}$ is defined by [9]:

$$
\boldsymbol{S}=\left[\begin{array}{c}
S_{0} \\
S_{1} \\
S_{2} \\
S_{3}
\end{array}\right]=\left[\begin{array}{c}
\Gamma_{x} \Gamma_{x}^{*}+\Gamma_{y} \Gamma_{y}^{*} \\
\Gamma_{x} \Gamma_{x}^{*}-\Gamma_{y} \Gamma_{y}^{*} \\
2 \operatorname{Re}\left(\Gamma_{x} \Gamma_{y}^{*}\right) \\
2 \operatorname{Im}\left(\Gamma_{x} \Gamma_{y}^{*}\right)
\end{array}\right]=\left[\begin{array}{c}
\Gamma_{x} \Gamma_{x}^{*}+\Gamma_{y} \Gamma_{y}^{*} \\
\Gamma_{x} \Gamma_{x}^{*}-\Gamma_{y} \Gamma_{y}^{*} \\
\Gamma_{x} \Gamma_{y}^{*}+\Gamma_{x}^{*} \Gamma_{y} \\
-i\left(\Gamma_{x} \Gamma_{y}^{*}-\Gamma_{x}^{*} \Gamma_{y}\right)
\end{array}\right]
$$

To obtain the evolution equation of the Stokes vector in a weakly anisotropic medium, the derivative of (4) has to be calculated with appropriate replacements of $\Gamma_{x}^{\prime}$ and $\Gamma_{y}^{\prime}$ from equation 3 . For example, for component $S_{1}$ such a calculation has the form:

$$
\begin{gathered}
S_{1}^{\prime}=\left(\Gamma_{x} \Gamma_{x}^{*}-\Gamma_{y} \Gamma_{y}^{*}\right)^{\prime}=\Gamma_{x}^{\prime} \Gamma_{x}^{*}+\Gamma_{x} \Gamma_{x}^{* *}-\Gamma_{y}^{\prime} \Gamma_{y}^{*}-\Gamma_{y} \Gamma_{y}^{* *}= \\
\frac{1}{2} i k \varepsilon_{0}^{-\frac{1}{2}}\left(v_{11} \Gamma_{x} \Gamma_{x}^{*}+v_{12} \Gamma_{y} \Gamma_{x}^{*}-v_{11}^{*} \Gamma_{x} \Gamma_{x}^{*}-v_{12}^{*} \Gamma_{x} \Gamma_{y}^{*}\right) \\
-\frac{1}{2} i k \varepsilon_{0}^{-\frac{1}{2}}\left(v_{21} \Gamma_{x} \Gamma_{y}^{*}+v_{22} \Gamma_{y} \Gamma_{y}^{*}-v_{21}^{*} \Gamma_{y} \Gamma_{x}^{*}-v_{22}^{*} \Gamma_{y} \Gamma_{y}^{*}\right)= \\
\frac{1}{2} i k \varepsilon_{0}^{-\frac{1}{2}}\left(\left(v_{11}-v_{11}^{*}\right) \Gamma_{x} \Gamma_{x}^{*}-\left(v_{22}-v_{22}^{*}\right) \Gamma_{y} \Gamma_{y}^{*}-\right. \\
v 21+\nu 12 * \Gamma x \Gamma y^{*}+v 12+v 21 * \Gamma y \Gamma x *
\end{gathered}
$$

For further calculations it is convenient to introduce fourcomponent complex vector $\boldsymbol{G}$ :

$$
\boldsymbol{G}=\left[\begin{array}{l}
G_{0} \\
G_{1} \\
G_{2} \\
G_{3}
\end{array}\right]=\left[\begin{array}{c}
-\frac{1}{2} i k \varepsilon_{0}^{-\frac{1}{2}}\left(v_{11}+v_{22}\right) \\
\frac{1}{2} i k \varepsilon_{0}^{-\frac{1}{2}}\left(v_{11}-v_{22}\right) \\
\frac{1}{2} i k \varepsilon_{0}^{-\frac{1}{2}}\left(v_{12}+v_{21}\right) \\
-\frac{1}{2} i k \varepsilon_{0}^{-\frac{1}{2}}\left(v_{12}+v_{21}\right)
\end{array}\right] .
$$

Using Eqs. (4) and (6), it is possible to rewrite (5) in the form:

$$
S_{1}^{\prime}=-\operatorname{Im}\left(G_{1}\right) S_{0}+\operatorname{Im}\left(G_{0}\right) S_{1}-\operatorname{Re}\left(G_{3}\right) S_{2}+\operatorname{Re}\left(G_{2}\right) S_{3} .
$$

The other $\boldsymbol{S}^{\prime}$ vector components are calculated in a similar way. Finally, the equation for the evolution of the Stokes vector can be written in the matrix form:

$$
\begin{gathered}
\mathbf{S}^{\prime}=\mathbf{M} \cdot \mathbf{S}= \\
{\left[\begin{array}{cccc}
\operatorname{Im}\left(G_{0}\right) & -\operatorname{Im}\left(G_{1}\right) & -\operatorname{Im}\left(G_{2}\right) & -\operatorname{Im}\left(G_{3}\right) \\
-\operatorname{Im}\left(G_{1}\right) & \operatorname{Im}\left(G_{0}\right) & -\operatorname{Re}\left(G_{3}\right) & \operatorname{Re}\left(G_{2}\right) \\
-\operatorname{Im}\left(G_{2}\right) & \operatorname{Re}\left(G_{3}\right) & \operatorname{Im}\left(G_{0}\right) & -\operatorname{Re}\left(G_{1}\right) \\
-\operatorname{Im}\left(G_{3}\right) & -\operatorname{Re}\left(G_{2}\right) & \operatorname{Re}\left(G_{1}\right) & \operatorname{Im}\left(G_{0}\right)
\end{array}\right] \cdot \mathbf{S},}
\end{gathered}
$$

where $\mathbf{M}$ is the Mueller matrix for a weakly anisotropic inhomogeneous medium and can be presented as a sum of three terms: $\mathbf{M}=\mathbf{M}_{\mathbf{a}}+\mathbf{M}_{\mathbf{d}}+\mathbf{M}_{\mathbf{b}}$. The first one, the attenuation component,

$$
\mathbf{M}_{\mathbf{a}}=\left[\begin{array}{cccc}
\operatorname{Im}\left(G_{0}\right) & 0 & 0 & 0 \\
0 & \operatorname{Im}\left(G_{0}\right) & 0 & 0 \\
0 & 0 & \operatorname{Im}\left(G_{0}\right) & 0 \\
0 & 0 & 0 & \operatorname{Im}\left(G_{0}\right)
\end{array}\right]
$$

describes isotropic attenuation common for all components of the Stokes vector. The second one, the dichroic term:

$$
\mathbf{M}_{\mathbf{d}}=\left[\begin{array}{cccc}
0 & -\operatorname{Im}\left(G_{1}\right) & -\operatorname{Im}\left(G_{2}\right) & -\operatorname{Im}\left(G_{3}\right) \\
-\operatorname{Im}\left(G_{1}\right) & 0 & 0 & 0 \\
-\operatorname{Im}\left(G_{2}\right) & 0 & 0 & 0 \\
-\operatorname{Im}\left(G_{3}\right) & 0 & 0 & 0
\end{array}\right],
$$

corresponds to the attenuation responsible for dichroism, that is, for selective attenuation of normal modes. The last term,

$$
\mathbf{M}_{\mathbf{b}}=\left[\begin{array}{cccc}
0 & 0 & 0 & 0 \\
0 & 0 & -\operatorname{Re}\left(G_{3}\right) & \operatorname{Re}\left(G_{2}\right) \\
0 & \operatorname{Re}\left(G_{3}\right) & 0 & -\operatorname{Re}\left(G_{1}\right) \\
0 & -\operatorname{Re}\left(G_{2}\right) & \operatorname{Re}\left(G_{1}\right) & 0
\end{array}\right]
$$

describes birefringence. Therefore real and imaginary parts of vector $\boldsymbol{G}$ correspond to hermitian and antihermitian parts of a permittivity tensor and are involving, respectively, isotropic attenuation or dichroism and birefringence. Please note that the quantity $\operatorname{Re}\left(G_{0}\right)$ does not appear in (7), which means that the total phase of the wave is lost in the Stokes vector evolution calculation. Only the phase difference between polarization modes can be retrieved from $\operatorname{Re}\left(G_{i}\right)$, where $\mathrm{i}=1,2,3$.

As an example, we will apply the obtained equations to the analysis of the Stokes vector evolution in nonrelativistic plasma in external magnetic field $\mathbf{B}$ with wave absorption due only to electron collisions (described by constant electron collision frequency $v_{c}$ ) and without a 
kinetic effect, such as occurs for ionosphere, laboratory or tokamak plasma. For a sufficient high electromagnetic wave frequency $\omega$ such plasma is weakly anisotropic and the components of the permittivity tenor transverse to the ray propagating in the $z$ direction have the form [10]:

$$
\left\{\begin{aligned}
\varepsilon_{x x} & =1-\frac{v\left((1+i s)^{2}-\bar{u} B_{x}^{2}\right)}{(1+i s)\left((1+i s)^{2}-u\right)} \\
\varepsilon_{x y} & =\frac{v\left(i(1+i s) \sqrt{\bar{u}} B_{z}+\bar{u} B_{x} B_{y}\right)}{(1+i s)\left((1+i s)^{2}-u\right)} \\
\varepsilon_{y x} & =\frac{v\left(-i(1+i s) \sqrt{\bar{u}} B_{z}+\bar{u} B_{x} B_{y}\right)}{(1+i s)\left((1+i s)^{2}-u\right)} \\
\varepsilon_{y y} & =1-\frac{v\left((1+i s)^{2}-\bar{u} B_{y}^{2}\right)}{(1+i s)\left((1+i s)^{2}-u\right)}
\end{aligned}\right.
$$

where $\bar{u}=\left(e / m_{e} c \omega\right)^{2}, u=\left(\omega_{c} / \omega\right)^{2}=\left(e B / m_{e} c \omega\right)^{2}$, $v=\left(\omega_{p} / \omega\right)^{2}=4 \pi e^{2} N_{e} / m_{e} \omega^{2}$ and $s=v_{c} / \omega$ are the standard parameters of plasma with density $N_{e}$. Weak anisotropy requires $u \ll 1$ or $v \ll 1$. Note that only one of the parameters $u$ or $v$ has to be small, while the other one can be comparable with unity. Assuming $\varepsilon_{0}=1-v$ as the isotropic part of the permittivity tensor (plasma without magnetic field and absorption), so from (1) $v_{i j}=\varepsilon_{i j}-(1-v) \delta_{i j}$, and weak dissipation in the plasma $(s \ll 1)$, the components of the Mueller matrix $\mathbf{M}$ from (6) and (11) in the first approximation of $s$ are:

$$
\begin{aligned}
& \operatorname{Im}\left(G_{0}\right) \\
& =-\frac{1}{2} k \varepsilon_{0}^{-1 / 2} \frac{v\left[-(3-u) \bar{u}\left(B_{x}^{2}+B_{y}^{2}\right)+2(1+u)\right]}{(1-u)^{2}} s \\
& \operatorname{Im}\left(G_{1}\right)=-\frac{1}{2} k \varepsilon_{0}^{-\frac{1}{2}} v \bar{u}\left(B_{x}^{2}-B_{y}^{2}\right) \frac{3-u}{(1-u)^{2}} s \\
& =-\Omega_{1} \frac{3-u}{1-u} s \\
& \operatorname{Im}\left(G_{2}\right)=-\frac{1}{2} k \varepsilon_{0}^{-\frac{1}{2}} v \bar{u} 2 B_{x} B_{y} \frac{3-u}{(1-u)^{2}} s=-\Omega_{2} \frac{3-u}{1-u} s \\
& \operatorname{Im}\left(G_{3}\right)=-\frac{1}{2} k \varepsilon_{0}^{-\frac{1}{2}} 4 v \sqrt{\bar{u}} B_{z} \frac{1}{(1-u)^{2}} s=-\Omega_{3} \frac{2}{1-u} s
\end{aligned}
$$

It is worth noting that in such a medium both isotropic and anisotropic attenuation are proportional to the collision frequency $v_{c}$ and inversely proportional to the electromagnetic wave frequency $\omega$.

$$
\begin{aligned}
& \begin{aligned}
\operatorname{Re}\left(G_{1}\right)=\frac{1}{2} k \varepsilon_{0}^{-\frac{1}{2}} v & \bar{u}\left(B_{x}^{2}-B_{y}^{2}\right) \frac{1}{1-u} \\
& =\Omega_{1} \sim 2.45 \cdot 10^{-11} \lambda^{3}\left(B_{x}^{2}-B_{y}^{2}\right) N_{e}
\end{aligned} \\
& \begin{aligned}
\operatorname{Re}\left(G_{2}\right)=\frac{1}{2} k \varepsilon_{0}^{-\frac{1}{2}} v \bar{u} 2 B_{x} B_{y} \frac{1}{1-u} \\
=\Omega_{2} \sim 2.45 \cdot 10^{-11} \lambda^{3}\left(2 B_{x} B_{y}\right)
\end{aligned} \\
& \operatorname{Re}\left(G_{3}\right)=\frac{1}{2} k \varepsilon_{0}^{-\frac{1}{2}} 2 v \sqrt{\bar{u}} B_{z} \frac{1}{1-u}=\Omega_{3} \sim 5.26 \cdot 10^{-13} \lambda^{2} B_{z}
\end{aligned}
$$

where coefficients $\Omega_{i}$, traditionally used in plasma polarimetry [8], correspond to the Cotton-Mouton effects $\left(\Omega_{1}\right.$ and $\left.\Omega_{2}\right)$ and to the Faraday phenomenon $\left(\Omega_{3}\right)$ $[11,12]$. In a nondissipative medium, $\operatorname{Im}\left(G_{i}\right)=0$ and $(7)$ reduce to the standard Stokes vector precession equation [8]:

$$
\mathbf{s}^{\prime}=\mathbf{\Omega} \times \mathbf{s} .
$$

To summarize, we have derived an evolution equation for the full four-component Stokes vector of the electromagnetic wave propagating in a weakly anisotropic, smoothly inhomogeneous medium, with no negligible absorption, as a function of permittivity tensor components. As a starting point we used the equations of a quasi-isotropic approximation (QIA), which follow in a consequent asymptotic way from Maxwell's equations. Obtained equation 7 allows to investigate the polarization state evolution in any medium with a sufficiently small combined parameter $\mu$, like different types of plasma (ionospheric, astrophysical, laboratory or tokamak plasma) or fibers.

This research outcome has been achieved under the research project No $1 / \mathrm{S} / \mathrm{KFiCh} / 14$ financed by the Ministry of Science and Higher Education subsidy for statutory activities.

\section{References}

[1] K.G.Budden, Radio Waves in the Ionosphere (Cambridge U. Press 1961).

[2] V.I.Ginzburg, Propagation of Electromagnetic Waves in Plasma (Gordon \& Breach 1970).

[3] Yu.A.Kravtsov, Sov. Phys. Dokl. 13, 1125 (1969).

[4] A.A. Fuki, Yu.A. Kravtsov, O.N. Naida, Geometrical Optics of Weakly Anisotropic Media (Gordon \& Breach, Lond., N.Y. 1997).

[5] Yu.A. Kravtsov, Yu.I. Orlov, Geometrical optics of inhomogeneous media (Springer Verlag, Berlin, Heidelberg 1990).

[6] Yu.A. Kravtsov et al., Physics-Uspekhi 39, 129(1996).

[7] F.De Marco, S.E.Segre, Plasma Phys. 14, 245 (1972).

[8] S.E.Segre, Plasma Phys. Control. Fusion 41, R57 (1999).

[9] M. Born, E. Wolf, Principles of Optics (Pergamon, Oxford 1980)

[10] B. Bieg et al., Physics Procedia 62, 102 (2015).

[11] B. Bieg et al., Nucl. Instr. Meth. Phys. Res. Sect. A 720, 157 (2013).

[12] S.E.Segre, J. Opt. Soc. Am. A 18, 2601 (2001). 